\title{
Nucleotide sequence of a cDNA encoding an $\alpha / \beta$-type gliadin from hexaploid wheat (Triticum aestivum)
}

\author{
Federico García-Maroto, ${ }^{1}$ Carmen Marañ, ${ }^{1,2}$ Francisco García-Olmedo ${ }^{1}$ and Pilar Carbonero ${ }^{1 *}$ \\ 'Cátedra de Bioquimica y Biologia Molecular, E.T.S. Ingenieros Agrónomos-UPM, E-28040 Madrid, \\ Spain (*author for correspondence); ${ }^{2}$ present address: Vrije Universiteit Brussel, Laboratory of Viral \\ Genetics, Paardenstraat 65, B-1640 St Genesius Rode, Belgium
}

The classification and nomenclature of wheat gluten proteins has been recently reassessed [6]. Gliadins of the $\alpha / \beta$ type are a subgroup of the $S$-rich prolamins which are characterized by a particular domain structure [6]. The cDNAs and/or genomic DNAs of a number of genetic variants have been cloned and sequenced $[1-5,7]$.

We have isolated a cDNA clone from a library obtained from developing endosperm of Triticum aestivum cv. Chinese Spring which encodes an $\alpha / \beta$-type gliadin that differs from previously described ones. The nucleotide sequence and deduced amino acid sequence of the new $\alpha / \beta$-gliadin clone, designated $\mathrm{MM} 1$, have been aligned in Fig. 1 with those corresponding to the class A-IV $\alpha / \beta$-gliadin of Okita et al. [3], which shows the closest resemblance to MMl among those previously described. The proline-rich $\mathrm{N}$-terminal domain is described in terms of six heptapeptide (VII-1 to VII-6) and six hexapeptide (VI-1 to VI-6) repeats, the hexapeptide consensus being contained in the heptapeptide one: (L) $\mathrm{P}_{Y}^{\mathrm{F}} \mathrm{PQPQ}$. The following features of gliadin MMI are different from the aligned class A-IV gliadin A735: (1) the additional heptapeptide VII-5, which is an identical replica of VII-3 and VII-4; (2) an additional nonapeptide in the first long poly-Q stretch (residues 141-149); (3) two QQQ deletions at positions 134 and 231. Apart from the alliuded insertions and deletions, only $13 \mathrm{nt}$ changes are observed out of 921 coding nt, 11 of which lead to amino acid changes.

\section{Acknowledgements}

F.G.M. was the recipient of a postdoctoral scholarship from the Ministerio de Educación y Ciencia. This work was supported by grant Bio88-0216 from Comisión Interministerial de Ciencia y Tecnología (Spain).

\section{References}

1. Anderson OD, Litts JC, Gautier M-F, Greene FC: Nucleic acid sequence and chromosome assignment of a wheat storage protein gene. Nucl Acids Res. 12: 8129-8144 (1984).

2. Kasarda DD, Okita TW, Bernardin JE, Baecker PA, Nimmo CC, Lew EJ-L, Dietler MD, Greene FC: Nucleic acid (cDNA) and amino acid sequences of $\alpha$-type gliadins from wheat (Triticum aestivum). Proc Natl Acad Sci USA 81: 4712-4716.

3. Okita TW, Cheesbrough C, Reeves CD: Evolution and heterogeneity of the $\alpha-\beta$-type and $\gamma$-type gliadin DNA sequences. J Biol Chem 260: 8203-82I3 (1985).

4. Rafalski JA, Scheets K, Metzler M, Peterson DM, Hedgcoth C, Sol] DG: Developmentally regulated plant genes: the nucleotide sequence of a wheat gliadin genomic clone. EMBO J 3: 1409-1415 (1984). 


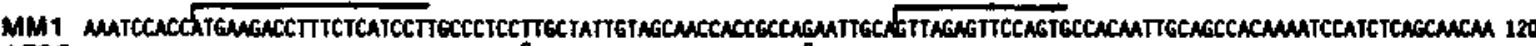
A735

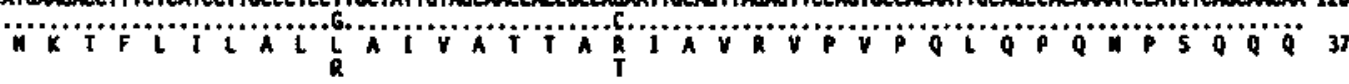
$\frac{V 11-1}{V 11-2} \quad V 1-1 \quad V 1-2 \longrightarrow V 1-3$

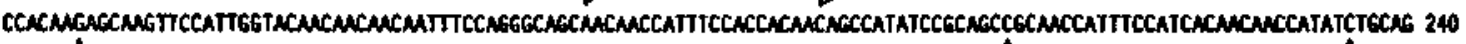

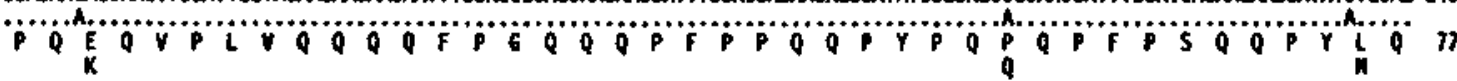

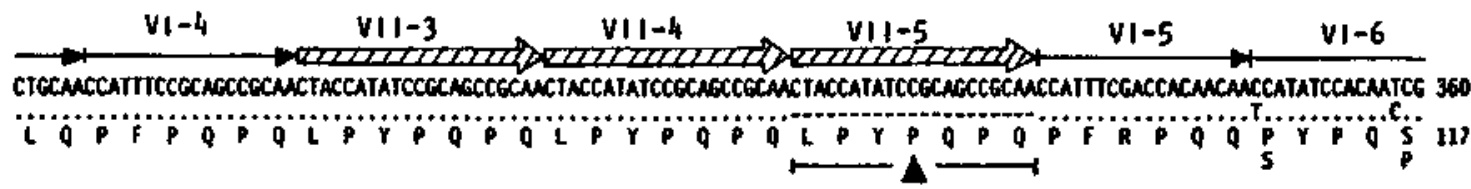
VII-6

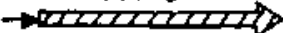

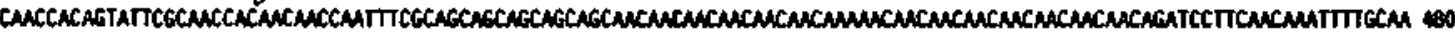
a p o i s

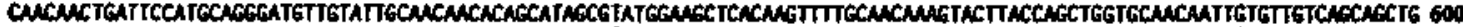

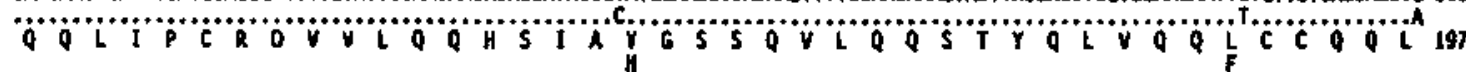

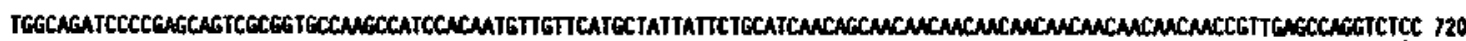
wö

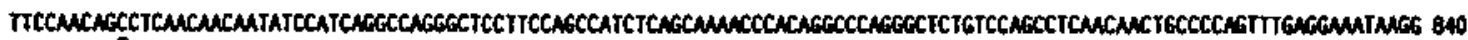

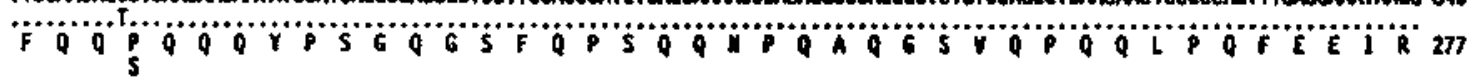

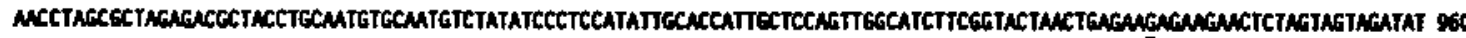

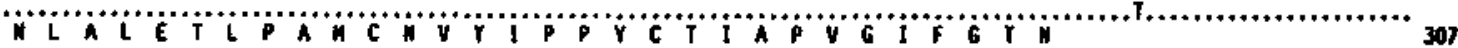
ATGATACACCGTTITCTIAGTCCATGGTTIGGTC SOA

Fig. 1. Nucleotide sequence and deduced amino acid sequence of $\alpha / \beta$-gliadin $M M 1$, aligned with those corresponding to class A.IV gliadin A735 [3]. Identical nt are indicated by dots ( $\cdot$ ) and missing nt by dashes (-). Heptapeptide (VII) and hexapeptide (VI) repeats are indicated by horizontal arrows. Amino acid stretches missing in A735 are indicated by horizontal bars (- $\Delta-$ ) and additional amino acid residues in $\mathrm{A} 735$ are printed out of line.

5. Reeves CD, Okita TW: Analyses of $\alpha / \beta$-type gliadin genes from diploid and hexaploid wheats. Gene 52: 257-266 (1987).

6. Shewry PR, Tatham AS, Forde J, Kreis M, Miflin BJ: The classification and nomenclature of wheat gluten proteins: A reassessment. J Cereal Sci 4: 97-106 (1986).
7. Sumner-Smith M, Rafalski JA, Sagiyama T, Stoll M, Söll $D$ : Conservation and variability of wheat $\alpha / \beta$-gliadin genes. Nucl Acids Res 13: 3905-3916 (1985). 